Supplemental information for:

\title{
Nanotextured dynamics of a light-induced phase transition in $\mathrm{VO}_{2}$
}

\author{
Aaron J. Sternbach ${ }^{1 * \dagger}$, Francesco L. Ruta ${ }^{1,2 \dagger}$, Yin Shi ${ }^{3}$, Tetiana Slusar ${ }^{4}$, Jacob Schalch ${ }^{5}$, \\ Guangwu Duan ${ }^{6}$, Alexander S. McLeod ${ }^{1}$, Xin Zhang ${ }^{6}$, Mengkun Liu ${ }^{7}$, Andrew J. Millis ${ }^{1}$, Hyun- \\ Tak Kim ${ }^{4}$, Long-Qing Chen ${ }^{3}$, Richard D. Averitt ${ }^{5}$, D. N. Basov ${ }^{1}$ \\ 1. Department of Physics, Columbia University, New York, NY, USA \\ 2. Department of Applied Physics and Applied Mathematics, Columbia University, New York, NY, USA \\ 3. Department of Materials Science and Engineering, The Pennsylvania State University, State College, PA, USA \\ 4. Electronics and Telecommunications Research Institute, Daejeon, South Korea \\ 5. Department of Physics, University of California San Diego, San Diego, CA, USA \\ 6. Department of Physics, Boston University, Boston, MA, USA \\ 7. Department of Physics, Stony Brook University, Stony Brook, NY, USA \\ $\dagger$ These authors contributed equally to this work \\ * Corresponding author. Email: as5049@columbia.edu
}




\section{Section S1: Properties of $\mathrm{VO}_{2}$ films on r-cut sapphire substrates}

Vanadium dioxide $\left(\mathrm{VO}_{2}\right)$ films of approximately $100 \mathrm{~nm}$ thickness were investigated in this work. A sharp 4-order of magnitude increase of the conductance is observed on a nominally similar thin film in the transition region, $338 \mathrm{~K}<\mathrm{T}<350 \mathrm{~K}$ (Fig.S1a). X-ray diffraction measurements reveal a dominant $(011)_{\mathrm{R}}$ phase above the transition indicating that these films are nearly $(>97 \%)$ singly oriented (Fig.S1b). Upon cooling, the film undergoes a symmetry lowering structural phase transition into a monoclinic (M1) phase. The possible orientations of the monoclinic phase are given by the established formula ${ }^{\mathrm{S} 1}$ :

$\left(\begin{array}{l}a \\ b \\ c\end{array}\right)_{M 1}=\left(\begin{array}{ccc}0 & 0 & 2 \\ 1 & 0 & 0 \\ 0 & 1 & -1\end{array}\right)_{R}$

This yields four possible orientations of the monoclinic structure, considering the equivalence of the $a$ and $b$ axes within the uniaxial rutile structure. As mentioned in the main text, two of these possible orientations, namely $(200)_{\mathrm{M} 1}$ and $(211)_{\mathrm{M} 1}$, are observed in our $\mathrm{VO}_{2}$ thin films that are nearly identical to the one we study in this work ${ }^{\mathrm{S} 2-\mathrm{S} 4}$.
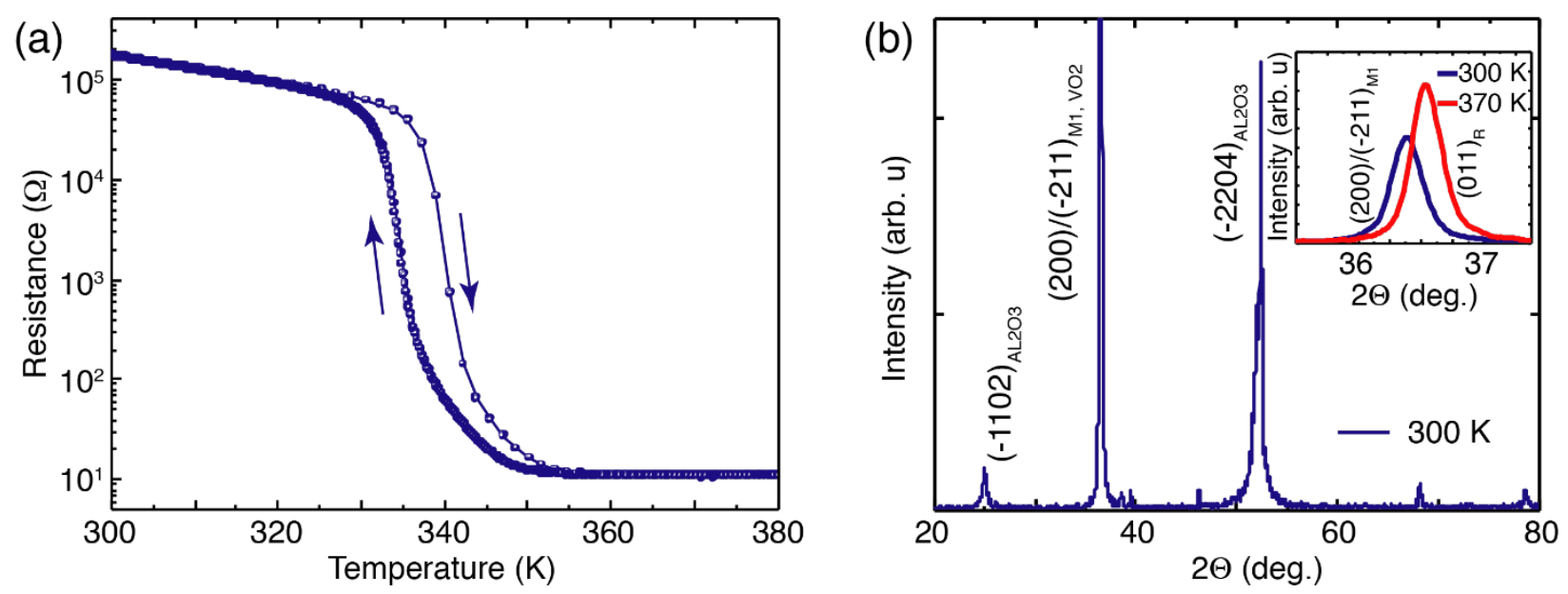

Figure S1: Characteristics of our $\mathrm{VO}_{2} / \mathrm{Al}_{2} \mathrm{O}_{3}$ sample. a, two-point resistance vs. temperature. $\mathbf{b}, \mathrm{XRD}$ intensity measured on a nominally similar film. 


\section{Section S2: Modeling the near-field signal of an arbitrarily oriented monoclinic $\mathrm{VO}_{2}$ thin}

film.

I. Calculating nano-optical spectra obtained in low symmetry crystals

Spectra of the near-field amplitude and phase of a laterally homogenous sample can be calculated given its complex Fresnel reflection coefficient, the geometric properties of the probe and sample, and the demodulation settings. Many analytical and numerical models have been developed to perform these calculations ${ }^{\mathrm{S} 5-\mathrm{S} 8}$. In this work, we utilize the lightning $\operatorname{rod} \operatorname{model}^{\mathrm{S} 5}$. This model assumes an axisymmetric hyperboloidal probe and planar axisymmetric sample geometry and is unique in that charge elements are rings of radius $R_{\tilde{z}}$, rather than monopoles or dipoles, emitting a field distribution with Fourier components weighted by first-order Bessel functions of the first kind $J_{0}$. The modulated near-field signal is calculated through a tapping cycle of the probe and demodulated with a sine transform of the back-scattered field. The linear charge density $\lambda_{Q}$ induced in the probe by incident illumination $E_{i n c}$ and by reflection off a planar sample a distance $\mathrm{d}$ below the tip is determined by solving a self-consistent scattering problem:

$\lambda_{Q}(q)=E_{\text {inc }} \Lambda_{0}(z)-\int_{0}^{\infty} d q \tilde{\lambda}_{Q}(q) q e^{-2 q d} r_{p}(q, \omega) \Lambda(\mathrm{q}, \mathrm{z})$

$\tilde{\lambda}_{Q}(q) \equiv \int_{0}^{L} d z^{\prime} \lambda_{Q}\left(z^{\prime}\right) e^{-q z^{\prime}} J_{0}\left(q R_{z^{\prime}}\right)$

where $\mathrm{q}$ is the in-plane momentum, $\omega$ is the frequency of light, $r_{p}$ is the sample Fresnel reflection coefficient under p-polarized illumination, $\mathrm{z}$ is a spatial coordinate along the length of the probe, and $\Lambda_{0}$ and $\Lambda$ are the illumination- and sample-induced probe response functions.

The lightning rod model is only strictly valid for planar structures of isotropic and nonbirefringent uniaxial materials. In low-symmetry materials exhibiting birefringence, like monoclinic $\mathrm{VO}_{2}$ for 
example, there will be a dependence on the in-plane polarization angle and some polarization rotation which violate the axisymmetry assumption. Therefore, we must make an ad-hoc approximation to model low-symmetry crystals. We suppose that the near-field signal for birefringent materials can be approximated by averaging the p-polarized reflection coefficients $r_{p}$ at different in-plane polarization angles $\phi$ and ignoring any s-polarized reflection $\left(r_{p s}=0, r_{p}=\right.$ $\left.r_{p p}\right)$. We rewrite Eq. (S2) accordingly:

$\lambda_{Q}(q) \approx E_{\text {inc }} \Lambda_{0}(z)-\frac{1}{2 \pi} \int_{0}^{\infty} \int_{0}^{2 \pi} d q d \phi \tilde{\lambda}_{Q}(q) q e^{-2 q d} r_{p}(q, \omega, \psi, \theta, \phi) \Lambda(\mathrm{q}, \mathrm{z})$

The Euler angles in the Tait-Bryan $\mathrm{X}_{1} \mathrm{Y}_{2} \mathrm{Z}_{3}$ convention, $\psi$ and $\theta$, orient the transformed axis $\hat{z}^{\prime}$ such that it is perpendicular to the reflection plane, while $\phi$ rotates the plane of incidence through the reflection plane. In this work, we are interested in the (200) and (211) orientations of an M1$\mathrm{VO}_{2}$ slab on a substrate. The Miller indices $(h k l)$ convey that the reflection plane intersects the crystal axes at $1 / h, 1 / k$, and $1 / l$ and can be converted to $\psi$ and $\theta$.

\section{Dielectric tensor of arbitrarily-oriented monoclinic $\mathrm{VO}_{2}$}

Calculating $r_{p p}(q, \omega, \psi, \theta, \phi)$ for the case of an arbitrarily-oriented monoclinic $\mathrm{VO}_{2}$ slab boils down to being able to express the dielectric tensor of $\mathrm{VO}_{2}$ in an arbitrary coordinate system specified by $\psi, \theta$, and $\phi$. The rest of the calculation will be straightforward and is discussed in subsection S2.III. 
(a)

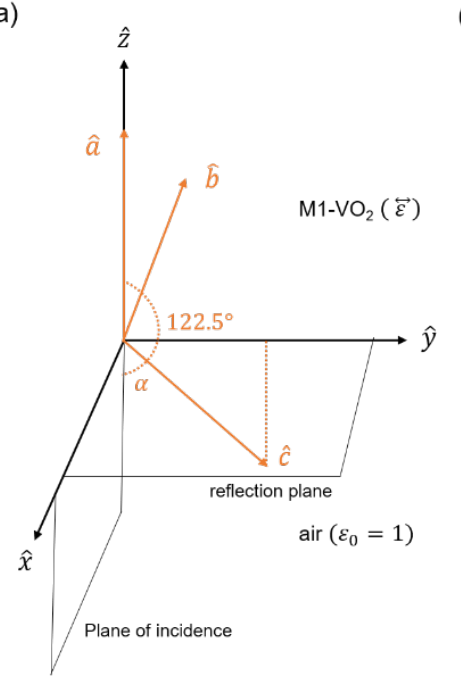

(b)

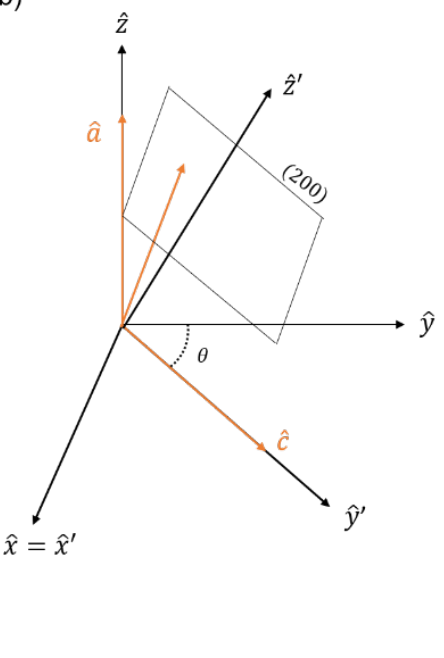

(c)

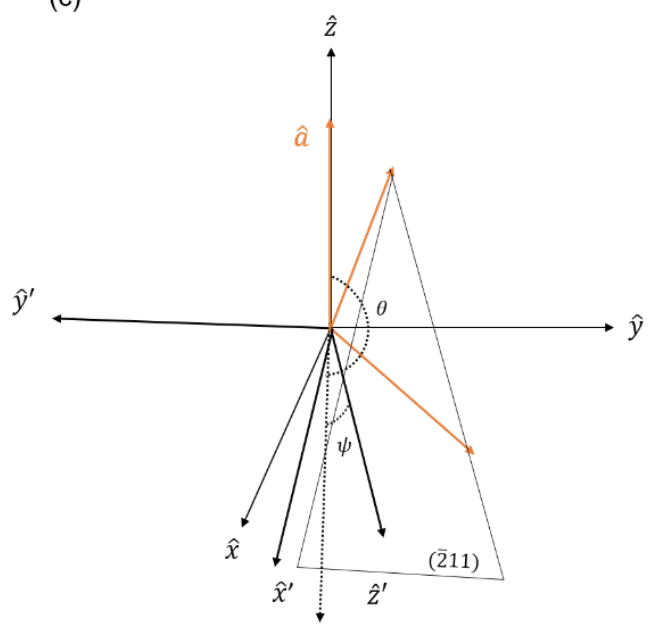

Figure S2: geometry. a, Reflection plane in the reference basis. b, Rotated Cartesian basis with $\hat{Z}^{\prime}$ perpendicular to the $(200)$ reflection plane. $\theta \approx 32.5^{\circ}$ and $\psi=0$. c, Rotated Cartesian basis with $\hat{Z}^{\prime}$ perpendicular to the (211) reflection plane. $\theta \approx 182.5^{\circ}$ and $\psi \approx-26.5$. Light is incident from air in the $x^{\prime} z^{\prime}$ plane onto the reflection plane formed by $x^{\prime} y^{\prime} . \phi$ controls the rotation of $\hat{x}^{\prime}$ and $\hat{y}^{\prime}$ about $\hat{z}^{\prime}$, thus rotating the in-plane polarization of incident light without changing the reflection plane.

We first define a reference orientation where $\psi=\theta=\phi=0$. We orient the axes such that the $a$ crystal axis is parallel to the $\hat{z}$ direction and $b$ lies along the $-\hat{x}$ direction. The $c$ crystal axis is in the yz plane at an angle $122.5^{\circ}$ from the $\hat{z}$ direction or equivalently $\alpha=57.5^{\circ}$ from the $-\hat{z}$ direction (Fig. S2a). The dielectric tensor of monoclinic $\mathrm{VO}_{2}$ will have the following form in the basis of $\hat{x}, \hat{y}$, and $\hat{z}$ :

$\overleftrightarrow{\varepsilon}(\omega)=\left[\begin{array}{ccc}\varepsilon_{b}(\omega) & 0 & 0 \\ 0 & \varepsilon_{y y}(\omega) & \varepsilon_{y z}(\omega) \\ 0 & \varepsilon_{y z}(\omega) & \varepsilon_{z z}(\omega)\end{array}\right]$

We assume that $\varepsilon_{y z} \approx 0$ and $\varepsilon_{z z}=\varepsilon_{a}(\omega) . \varepsilon_{y y}$ was a frequency-independent fitting parameter.

With these assumptions, the dielectric tensor in the reference basis can be simplified:

$\overleftrightarrow{\varepsilon}(\omega)=\left[\begin{array}{ccc}\varepsilon_{b}(\omega) & 0 & 0 \\ 0 & \varepsilon_{y y} & 0 \\ 0 & 0 & \varepsilon_{a}(\omega)\end{array}\right]$ 
The dielectric tensor can be written for any arbitrary crystallographic orientation by rotating the basis. To perform the rotation, we first express the Miller indices (200) and (211) as Euler angles. In monoclinic materials like $\mathrm{VO}_{2}$, the Miller indices are expressed in a non-orthogonal crystal basis and should be transformed to the orthogonal xyz basis before converting to Euler angles. Once we have Euler angles, the matrix $R$ in Equation $\mathrm{S} 7$ can be used to change the basis of the dielectric tensor to the appropriate reflection plane as shown in Fig. S2:

$\overleftrightarrow{\varepsilon}(\omega)=R^{-1} \overleftrightarrow{\varepsilon}(\omega) R$

$R=\left[\begin{array}{ccc}\cos \psi \cos \phi & \cos \theta \sin \phi+\sin \theta \sin \psi \cos \phi & \sin \theta \sin \phi-\cos \theta \sin \psi \cos \phi \\ -\cos \psi \sin \phi & \cos \theta \cos \phi-\sin \theta \sin \psi \sin \phi & \sin \theta \cos \phi+\cos \theta \sin \psi \sin \phi \\ \sin \psi & -\sin \theta \cos \psi & \cos \theta \cos \psi\end{array}\right]$

III. Fresnel reflection coefficient of an arbitrary anisotropic crystal slab on a substrate

With the dielectric tensor in the rotated basis, the complex reflection coefficient of p-polarized incident light $r_{p}(q, \omega)$ can be easily calculated for a slab of arbitrarily-oriented $\mathrm{VO}_{2}$ using the $4 \times 4$ propagation matrix method ${ }^{\mathrm{S} 9}$. Maxwell's curl equations in an arbitrary anisotropic medium can be written compactly in the form of a first-order system of differential equations (in SI units):

$\frac{\partial \Psi}{\partial z^{\prime}}=i \Delta \Psi$

where $\Psi=\left[\begin{array}{llll}\sqrt{\epsilon_{0}} E_{x} & \sqrt{\mu_{0}} H_{y} & \sqrt{\epsilon_{0}} E_{y} & -\sqrt{\mu_{0}} H_{x}\end{array}\right]^{T}$ and the components of the $\Delta$-matrix are as follows when the xz plane is the plane of incidence: 
$\Delta=\frac{\omega}{c}\left[\begin{array}{cccc}-\varepsilon_{z x}^{\prime} q c / \omega \varepsilon_{z z}^{\prime} & 1-q^{2} c^{2} / \omega^{2} \varepsilon_{z z}^{\prime} & -\varepsilon_{z x}^{\prime} q c / \omega \varepsilon_{z z}^{\prime} & 0 \\ \varepsilon_{x x}^{\prime}-\varepsilon_{x z}^{\prime} \varepsilon_{z x}^{\prime} / \varepsilon_{z z}^{\prime} & -\varepsilon_{x z}^{\prime} q c / \omega \varepsilon_{z z}^{\prime} & \varepsilon_{x y}^{\prime}-\varepsilon_{x z}^{\prime} \varepsilon_{z y}^{\prime} / \varepsilon_{z z}^{\prime} & 0 \\ 0 & 0 & 0 & 1 \\ \varepsilon_{y x}^{\prime}-\varepsilon_{y z}^{\prime} \varepsilon_{z x}^{\prime} / \varepsilon_{z z}^{\prime} & -\varepsilon_{y z}^{\prime} q c / \omega \varepsilon_{z z}^{\prime} & \varepsilon_{y y}^{\prime}-q^{2} c^{2} / \omega^{2}-\varepsilon_{y z}^{\prime} \varepsilon_{z y}^{\prime} / \varepsilon_{z z}^{\prime} & 0\end{array}\right]$

The general solution to the system of differential equations in equation S1 that gives the electromagnetic field components after traveling a distance $d$ inside the medium is:

$\Psi\left(z^{\prime}+d\right)=\exp (i \Delta d) \Psi\left(z^{\prime}\right)$

The matrix $P(d)=\exp (i \Delta d)$ is called the propagation matrix. To evaluate the exponential of a matrix, we compute its eigenvalues $k_{z 1}, k_{z 2}, k_{z 3}, k_{z 4}$ and eigenvectors $V$.

$$
P(d)=V\left[\begin{array}{cccc}
\exp \left(\mathrm{i} k_{z 1} d\right) & 0 & 0 & 0 \\
0 & \exp \left(\mathrm{i} k_{z 2} d\right) & 0 & 0 \\
0 & 0 & \exp \left(\mathrm{i} k_{z 3} d\right) & 0 \\
0 & 0 & 0 & \exp \left(\mathrm{i} k_{z 4} d\right)
\end{array}\right] V^{-1}
$$

The eigenvalue problem was evaluated numerically at every frequency using the NumPy linear algebra library in Python.

Finally, the p-polarized reflection coefficient of the p-polarized incident light $r_{p p}$ can be calculated from the continuity requirements at the interfaces. The incident and exit media are air and $\mathrm{Al}_{2} \mathrm{O}_{3}$ in our case, to which we assign isotropic refractive indices of 1 and $n_{t}$, respectively. The $\mathrm{Al}_{2} \mathrm{O}_{3}$ dielectric function is taken from Ref. [S10]. Polarization rotation upon reflection was neglected $\left(r_{p s}=0\right)$.

$r_{p p}=\frac{a_{1} a_{6}-a_{3} a_{4}}{a_{3} a_{5}-a_{2} a_{6}}$

where: 


$$
\begin{aligned}
& a_{1,2}=n_{t} P_{12}-\cos \gamma_{t} P_{12} \pm \cos \gamma_{i}\left(n_{t} P_{11}-\cos \gamma_{t} P_{21}\right) \\
& a_{3}=n_{t} P_{13}-\cos \gamma_{t} P_{23}-\cos \gamma_{i}\left(n_{t} P_{14}-\cos \gamma_{t} P_{24}\right) \\
& a_{4,5}=n_{t} \cos \gamma_{t} P_{32}-P_{42} \pm \cos \gamma_{i}\left(n_{t} \cos \gamma_{t} P_{11}-P_{41}\right) \\
& a_{6}=n_{t} \cos \gamma_{t} P_{33}-P_{43}-\cos \gamma_{i}\left(n_{t} \cos \gamma_{t} P_{34}-P_{44}\right)
\end{aligned}
$$

and $\cos \gamma_{i, t}=\sqrt{1-\left(q c / n_{i, t} \omega\right)^{2}}$ are cosines of the angles of incidence and transmission, accordingly.

\section{Lorentz oscillator parameters for $\mathrm{M} 1-\mathrm{VO}_{2}$ dielectric functions}

The dielectric functions of $\mathrm{M} 1-\mathrm{VO}_{2}$ were studied in Ref. [S11] with far-field reflectometry. The dielectric functions are parametrized by the Lorentz oscillator model:

$\varepsilon(\omega)=\varepsilon_{\infty}+\sum_{i=1}^{N} \frac{\omega_{0, i}^{2} s_{i}^{2}}{\omega_{0, i}^{2}-\omega^{2}-i \Omega \omega}$

Where the static dielectric function is described by $\varepsilon_{\infty}$ while the oscillator strength $s_{i}^{2}$, center frequency, $\omega_{0, i}^{2}$ and linewidth govern $\Omega$ the optical response of a particular phonon. The subscript $\mathrm{i}$ is used to indicate the relevant phonon-resonance. The oscillator parameters from Ref. [S11] were used in this work, except for the high-frequency permittivities $\varepsilon_{a, \infty}$ and $\varepsilon_{b, \infty}(\sim 12$ in Ref. [S11]). We set values for $\varepsilon_{a, \infty}=\varepsilon_{b, \infty}=\varepsilon_{a b, \infty}$, as well as for $\varepsilon_{y y, \infty}$, by roughly fitting the model to the experimental data. The oscillator parameters are summarized in Table S1 below.

Table S1: Lorentz oscillator parameters for the dielectric functions of $\mathrm{M} 1-\mathrm{VO}_{2}$ along its $a$ and $b$ crystal axes. The $\omega_{0, i}, s_{i}$, and $\gamma_{i}$ parameters were taken from Ref. [S11]. The $\epsilon_{\infty}$ parameters are 
extracted from fitting the nano-FTIR data in this work. The static contribution to the $\varepsilon_{y y}$ component of the dielectric tensor was set arbitrarily to 5 .

\begin{tabular}{|l|l|l|l|l|l|l|l|l|}
\hline & & & & & & & & \\
\hline $\mathrm{i}$ & $\omega_{0, i}\left(\mathrm{~cm}^{-1}\right)$ & \multicolumn{1}{|c|}{$s_{i}$} & $\gamma_{i}$ & $\epsilon_{a b, \infty}$ & $\omega_{0, i}\left(\mathrm{~cm}^{-1}\right)$ & $S_{i}$ & $\gamma_{i}$ & $\epsilon_{y y, \infty}$ \\
\hline 1 & 189 & 0.54 & 0.012 & 9 & 277 & 4.01 & 0.062 & 5 \\
\hline 2 & 281 & 4.53 & 0.074 & - & 324 & 3.49 & 0.038 & - \\
\hline 3 & 310 & 6.69 & 0.055 & - & 351 & 1.67 & 0.041 & - \\
\hline 4 & 336 & 0.49 & 0.023 & - & 367 & 1.88 & 0.044 & - \\
\hline 5 & 500 & 0.77 & 0.060 & - & 392 & 0.99 & 0.038 & - \\
\hline 6 & 521 & 1.34 & 0.047 & - & 519 & 1.08 & 0.110 & - \\
\hline 7 & 607 & 3.42 & 0.040 & - & 709 & 0.25 & 0.071 & - \\
\hline 8 & 637 & 0.67 & 0.100 & - & - & - & - & - \\
\hline 9 & 720 & 0.15 & 0.056 & - & - & - & - & - \\
\hline
\end{tabular}

\section{Section S3: Image correlations}

Correlations between the images of 'electronic' and 'phonon' enhanced nano-optical contrasts (Fig.3) were analyzed as follows. An image of the near-field amplitude collected at $\omega=715 \mathrm{~cm}^{-1}$ and $\mathrm{T}=335 \mathrm{~K}$ was taken to represent the 'phonon' enhanced nano-optical contrast (Fig.S3a) while an image taken at $\omega=1,000 \mathrm{~cm}^{-1}$ and $\mathrm{T}=338 \mathrm{~K}$ was taken to represent the 'electronic' nano-optical contrast (Fig.S3b), as described in the main text. The histograms of these images (Fig.S3d and S3e) were fit with a function of the form:

$V=\sum_{1}^{N} \exp \left(-\left(\frac{S-S_{c}}{S_{w}}\right)^{2}\right)$

A reasonably good fit to the 'electronic' data requires at least two terms $(\mathrm{N}=2)$ in Eq. (S18), which are taken to represent the insulating and metallic distributions. The threshold separating the insulating and metallic domains is chosen as the intersection of the two Gaussians $(\mathrm{S}=0.61$ in Fig. $\mathrm{S} 3 \mathrm{e})$ which is a common procedure ${ }^{\mathrm{S} 12}$. The images are then binarized where pixels with higher and lower intensities than the threshold are taken to be metallic and insulating respectively. The 
'phonon' contrast images (Fig. S3a) are well described with a single term (N=1) in equation (S18), see Fig. S3d. We speculate that the single distribution may be impacted by the approximately $45-$ degree angle of the twin interface with the substrate normal ${ }^{\mathrm{S} 3}$, leading to a continuous variation in the near-field signal across the interface. The probability for the domains to nucleate at twin interfaces $\left(95_{-11}^{+5} \%\right)$ is calculated with the threshold at the mean value of the distribution. The error is estimated by calculating the number of emergent domains with the thresholds used to binarize the 'phonon' contrast taken at the red and blue lines shown in Fig. S3d.

The nano-optical contrast is sensitive to the heterogeneity of the dielectric tensor. Correspondingly, if the parameters within the Lorentz oscillator model depend on the spatial coordinate, we anticipate that spatially textured nano-optical contrast can be observed. While we do not anticipate significant local variations of the fundamental oscillator strength or linewidth of phonons, strain can produce a variation of phonon's resonant frequencies ${ }^{\mathrm{S} 1, \mathrm{~S} 13}$. The magnitude of the frequency shift is described by the Grüneisen parameter, $\gamma_{i}=-\frac{V}{\omega_{0, i}} \frac{\partial \omega_{0, i}}{\partial V}$, where $\mathrm{V}$ is the unstrained volume, the subscript $i$ indicates the relevant vibrational mode, and $\omega_{0, i}$ is the centerfrequency of this vibrational mode. The application of uni-axial strain, therefore, produces a monotonic variation in the center frequency of the vibrational mode. Uni-axial strain along the caxis of $\mathrm{VO}_{2}$ is also known to produce a monotonic change of the transition temperature ${ }^{\mathrm{S} 14}$.

Nucleation of metallic domains is observed at contrast interfaces (Fig. 3 of the main text). If the observed room-temperature contrast were associated with strain we, therefore, expect the lowest transition temperatures, and the highest compressive strain, present within the system would exist at contrast interfaces. Thus, if we are to satisfy the hypothesis that strain is responsible for the observed contrast, it should be possible for the near-field amplitude to either increase or decrease on either side of the interface as the local compressive strain is relaxed. In Fig. S3f we 
show the near-field amplitude calculated at the probe frequency of $710 \mathrm{~cm}^{-1}$ as a function of the resonant energy of the $A_{u}$ phonon mode, which has a resonant frequency of $607 \mathrm{~cm}^{-1}$ in the unstrained case $^{\mathrm{S} 10}$ (black arrow in Fig. S3f). Note that the other phonon modes have a negligible impact on $S$ at this probe frequency. We find that $S$ depends strongly on $\omega_{0}$ and is non-monotonic for blueshifts exceeding $+60 \mathrm{~cm}^{-1}$. Thus, one possibility is that compressive strain is associated with blueshifts of the monoclinic phonon, which would allow $S$ to either increase or decrease along with a monotonic decrease of $\omega_{0}$. Yet, when attempting to satisfy the hypothesis that strain is responsible for the observed contrast two issues are encountered. First, while an increase of $S$ is readily expected from a smooth decrease of $\omega_{0}$, producing a decrease of $S$ would require an abrupt decrease of $\omega_{0}$. Such an erratic dependence of $\omega_{0}$ on the real-space coordinate is unlikely. Second, prior works show compressive strain, and the lowest transition temperatures, are associated with redshifts of the phonon frequency ${ }^{\mathrm{S} 1, \mathrm{~S} 13}$. Thus, we conclude that strain is unlikely to fully account for the observed room-temperature contrast.

Next, we discuss nucleation at twin interfaces. A detailed assessment of twin interface nucleation energy has been discussed in Ref. [S15]. First, there is an energetic cost that is needed to maintain a twin interface, $\gamma_{21}$. We denote the energy cost to maintain an interface between rutilemetal and monoclinic insulating states, $\gamma_{\mathrm{RM}}$. If a metallic domain were to form in a region with purely $(200)_{\mathrm{M} 1}$ or $(\underline{2} 11)_{\mathrm{M} 1}$ orientation, these energy costs would add giving rise to the total interfacial energy, $\gamma=\gamma_{\mathrm{RM}}+\gamma_{21}$. However, if the rutile metal domain were to form at a twin interface, the symmetry raising transition causes both sides of the interface to acquire the same rutile $(011)_{\mathrm{R}}$ structure. Thus, the energy cost associated with the twin interface can be eliminated for interface dominated nucleation giving rise to lower interfacial energy, $\gamma=\gamma_{\mathrm{RM}}-\gamma_{21}$. We stress that the cartoon picture we present above is simplified, as twin interfaces can host numerous 
complex phenomena that locally alter the energetic landscape. These include local variations in strain $^{\mathrm{S1}}$, impurities, defects and local changes of stoichiometry ${ }^{\mathrm{S} 16, \mathrm{S17}}$. Grain boundaries, that are observed in our film, add further complexity. A complete account of all bulk and local energetic costs is beyond the scope of this work. Independent of all factors that influence the local energetics our experimental data supports the conclusion that nucleation proceeds from twin interfaces.

The $607 \mathrm{~cm}^{-1} \mathrm{~A}_{\mathrm{u}}$ phonon drives the $b$-axis component of the permittivity of $\mathrm{VO}_{2}$ negative in the frequency range of $607 \mathrm{~cm}^{-1} \lesssim \omega \lesssim 800 \mathrm{~cm}^{-1}$. Our modeling corroborates the notion that nearfield contrast is strongly enhanced by phonons reaching a contrast sensitivity that is $7-100 \mathrm{x}$ higher than observed outside of the Reststrahlen band (Fig. S3g and S3h). At frequencies away from the Reststrahlen band, the calculated nano-optical contrasts are below our noise level and near-field images will appear homogeneous, consistent with our observations (Figs. $2 \mathrm{~d}$ and $2 \mathrm{e}$ of the main text).
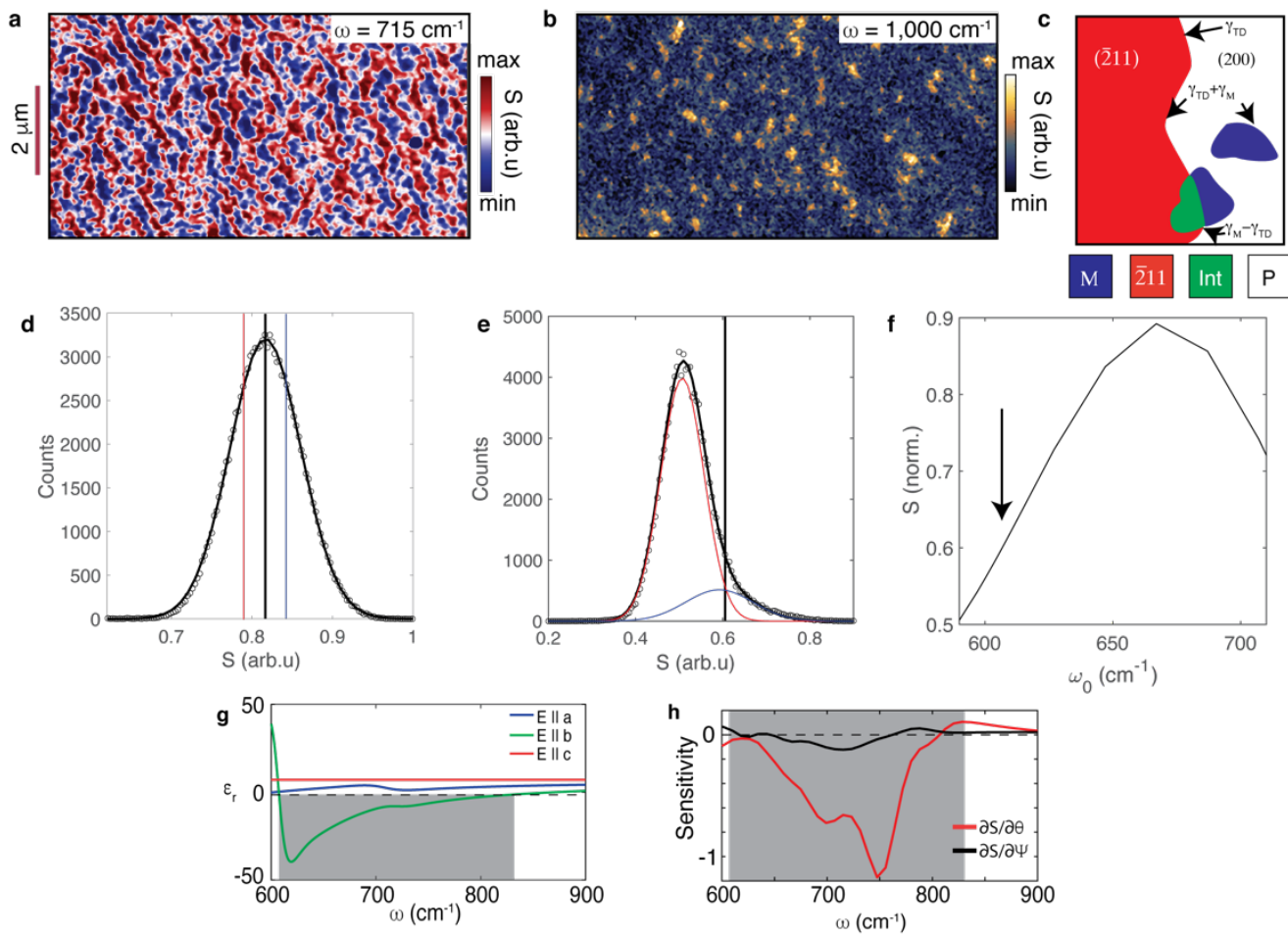

Figure S3: Imaging Correlations and sensitivity. a, Data showing the near-field amplitude, $S$, recorded at the frequency $\omega=715+/-20 \mathrm{~cm}^{-1}$ at T=335 K, below the onset of the transition region of the IMT. b, Data showing $S$ 
recorded at the frequency $\omega=1,000 \mathrm{~cm}^{-1}$ at $\mathrm{T}=338 \mathrm{~K}$, which is at the onset of the transition region of the IMT. The images in panels (a) and (b) are co-located. c, a cartoon schematic representing the interfacial energy cost associated with forming rutile-metallic domains. The colors used in the figure are: white for the parent $(\mathrm{P})$ insulating $(200)_{\mathrm{M} 1}$ oriented monoclinic regions; red for the (211) $)_{\mathrm{M} 1}$ oriented monoclinic regions; blue for rutile-metal within an initially $(200)_{\mathrm{M} 1}$ oriented region; green rutile metal within an initially $(211)_{\mathrm{M} 1}$ oriented region. The twin-domain interface is associated with the energy cost $\gamma_{\mathrm{TD}}$. The interfacial energy cost associated with a metal-insulator boundary is given by $\gamma_{\text {M. }} \mathbf{d}$, histogram of the data in panel (a). The binary data presented in the main text used a threshold at the black line. Thresholds at the red and blue lines were used to calculate the error presented in the main text. e, Histogram of the data in panel (b). The threshold for binarization was taken at the black line while the thresholds at the red and blue lines were used to determine the error, as stated in the text. f, Calculation of the near-field amplitude, $S$, after artificially shifting the center frequency of the $607 \mathrm{~cm}^{-1} \mathrm{~A}_{u}$ phonon mode to the variable frequency $\omega_{0}$. g, Principal values of the dielectric tensor used in our calculations. $\mathbf{h}$, Contrast sensitivity to the local structural orientation, defined by the Euler angles $\theta$ and $\Psi$ (see Section S2). The region where the $b$-axis of the dielectric tensor becomes negative is indicated with gray shading in panels $(\mathbf{g})$ and $(\mathbf{h})$. 


\section{Section S4: Quantitative assessment of nucleation and growth rates}

To quantify rates of nucleation and growth in $\mathrm{VO}_{2}$ we analyzed a series of images collected at a series of time delays, $\Delta \mathrm{t}$, following photoexcitation with sufficient fluence to trigger the IMT. As explained in the main text the full width at half maximum (FWHM) of the autocorrelation function (ACF) increases along with increasing $\Delta \mathrm{t}$ (Fig. 4 of the main text). Thus, real space growth is directly observed. Note that ACF data shown in Fig. 4e were computed from transient nanoimaging data acquired over a larger field of view $(6 \times 6 \mu \mathrm{m})$ than the transient imaging data shown in Figs. 1, 4a, and $4 \mathrm{~b}$ of the main text, for improved statistics. To quantify the fractional area of transient metal (Fig. 4 of the main text) one must determine the number of domains, in addition to their size. Given the complexity of the observed nanotexture, we preferred the following approach, which minimizes the number of assumptions needed to analyze the data. The data were binarized in a similar manner described in section S3. Namely histograms of the transient amplitude, such as the representative example shown in Fig. $4 \mathrm{c}$ of the main text, were fit with a bimodal distribution Eq. (S18). The intersection of the upper and lower distributions was considered to be the threshold. The number of binarized pixels is used to determine the transient area. In Fig. S4 we compare the transient area extracted from binarization with the ACF domain size (Fig. 4) after multiplying the ACF domain size by an arbitrary constant perfector. The two analyses yield consistent results: namely (1) the procedures for extracting these areas both show a monotonic increase of the area of transient metal along with the time delay and (2) the same conclusion of boundary driven nucleation is obtained independent of the procedure used to extract the area.

The growth of the emergent metal has different rate laws depending on where the metal nucleates.

J. W. Cahn has deduced the growth rate laws for a new phase nucleating in the bulk randomly, at 
the edges of the grain boundaries, and the corners of the grain boundaries in three-dimensional systems in Ref. [S18]. Since here we are investigating the surface of the material, we adapted Cahn's method to deduce the similar rate laws in two dimensions,

$X_{r}^{2 d}\left(\tau_{r}\right)=1-\exp \left[-f_{r}^{2 d}\left(\tau_{r}\right) / \gamma_{r}^{2 d}\right](\mathrm{S} 19 \mathrm{a})$

$X_{e}^{2 d}\left(\tau_{e}\right)=1-\exp \left[-f_{e}^{2 d}\left(\tau_{e}\right) / \gamma_{e}^{2 d}\right](\mathrm{S} 19 \mathrm{~b})$

$X_{c}^{2 d}\left(\tau_{c}\right)=1-\exp \left[-f_{c}^{2 d}\left(\tau_{c}\right) / \gamma_{c}^{2 d}\right](\mathrm{S} 19 \mathrm{c})$

where $X_{r}^{2 d}, X_{e}^{2 d}$, and $X_{c}^{2 d}$ are the metal fraction on the surface of the cases where the metal nucleates on the surface randomly, at the edges of the grain boundaries on the surface, and the corners of the grain boundaries on the surface, respectively. $\tau$ 's are dimensionless variables proportional to the time $t$ and $\gamma$ 's are dimensionless constants related to rates of metal nucleation and growth. The function $f$ is given by:

$f_{r}^{2 d}\left(\tau_{r}\right)=\tau_{r}^{3}(\mathrm{~S} 20 \mathrm{a})$

$f_{e}^{2 d}\left(\tau_{e}\right)=\tau_{e} \int_{0}^{1}\left[1-\exp \left(-\tau_{e}^{2}\left(\sqrt{1-x^{2}}-x^{2} \ln \frac{1+\sqrt{1-x^{2}}}{x}\right)\right)\right] d x$

$f_{c}^{2 d}\left(\tau_{c}\right)=\tau_{c}^{2}-2 \tau_{c}+2\left[1-\exp \left(-\tau_{c}\right)\right]$

which are plotted with log scale in Fig. S4 (in this way the curves only displace horizontally as the proportionality of $\tau$ 's to $t$ changes). For the case of homogeneous nucleation, we have:

$\tau_{r}=\left(I_{r} G^{2}\right)^{1 / 3} \Delta t$

$\gamma_{r}^{2 d}=\frac{\pi}{3}$

where $I_{r}$ is the homogeneous nucleation rate per unit area. In the case of corner driven nucleation we have:

$\tau_{c}=I_{c} \Delta t$

$\gamma_{c}^{2 d}=\frac{I_{c}^{2}}{\pi C G^{2}}$ 
where $I_{C}$ is the corner nucleation rate per corner and $C$ is the grain corner number per unit area. Finally in the case that of edge driven nucleation, discussed in the main text, we have:

$\tau_{e}=\sqrt{I_{e} G} \Delta t \quad(\mathrm{~S} 23 \mathrm{a})$

$\gamma_{e}^{2 d}=\frac{1}{2 L} \sqrt{\frac{I_{e}}{G}}(\mathrm{~S} 23 \mathrm{~b})$

where $I_{e}$ is the nucleation rate at grain edges per unit length, $G$ is the growth rate, and $\mathrm{L}$ is the grain edge length per unit area. In the main text we take $L \sim 4 / D$ where $\mathrm{D}$ is the average size of the grains. Comparison with the experimental data reveals agreement only with the case of nucleation at the edges of the grain boundaries on the surface, $f_{e}^{2 d}\left(\tau_{e}\right)$, demonstrating that the light-induced nucleation of the metal occurs primarily at the edges of the grain boundaries. 


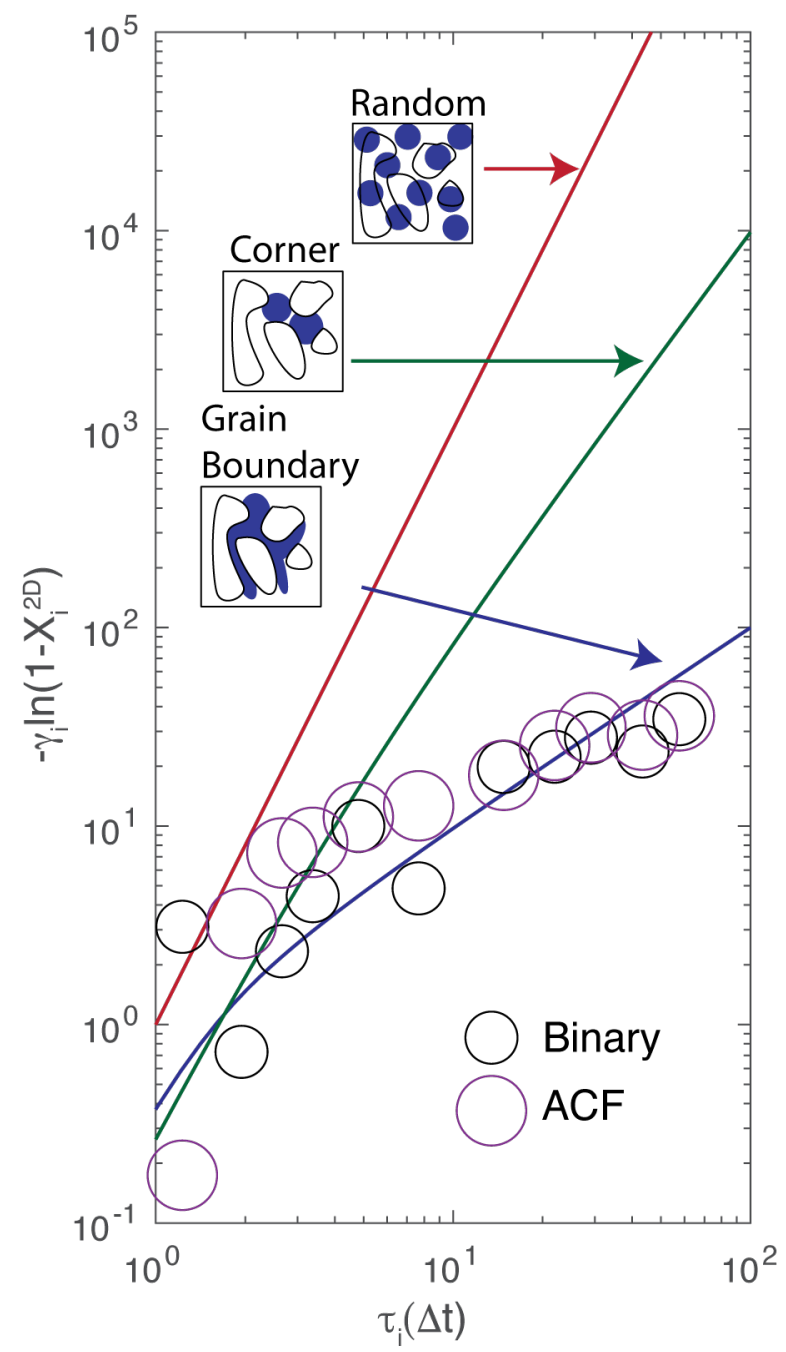

Figure S4: rate of growth. The fractional area of transient metal, $X_{i}^{2 D}$, is quantified by the methods described in the Supplementary text. The scaled area $-\gamma_{i} \ln \left(1-X_{i}^{2 D}\right)$ is plotted against the scaled time-delay $\tau_{i}(\Delta t)$ and compared with theoretical curves for the case of random (red), corner driven (green), and grain boundary driven (blue) nucleation (see Supplementary Section S4). The insets show cartoons where the black perimeters indicate grain boundaries and blue-filled regions indicate metallic patches. 


\section{Section S5: Anchoring the IMT with antenna launchers}

To locally enhance the electric field of the pump, we patterned a series of antennas on the $\mathrm{VO}_{2}$ thin film. Enhancement of $\Delta \mathrm{S}$ was consistently observed surrounding the antennas (Fig. S5a and S5c). Data within the white dashed rectangle indicated in Fig.S5a are shown in the Fig.5 of the main text for a series of pump-probe time delays. FTDT simulations were carried out in Lumerical to numerically determine the local electric field in the vicinity of the antenna. Here, the dielectric constant of $\mathrm{VO}_{2}$ in the insulating state was assumed to be isotropic with a value of $\varepsilon=8$ (see section 2). We checked a series of values for $\mathrm{VO}_{2}$ and found that values of the permittivity within the range of $\varepsilon=4-12$ resulted in similar results to those shown in Fig.S5b. The gold was taken to have the experimentally determined height of $80 \mathrm{~nm}$ while the $\mathrm{VO}_{2}$ thin film was taken to have a $100 \mathrm{~nm}$ thickness. The antennas have lengths of $1 \mu \mathrm{m}$ and widths of $500 \mathrm{~nm}$. The material constants of gold were used to describe the antennas in our simulations. The pump wavelength of $1.03 \mu \mathrm{m}$ was used to match the experiment. Further, we took the source to be a plane wave with ppolarization incident at $\theta=30$ degrees from the sample normal and a $\phi=40$-degree angle from the $\mathrm{X}$-axis (see inset in Fig.S5b), which is approximately the case used in the experimental results reported in Fig.5 and Fig.S5. The good agreement between the measured nano-texture of $\Delta \mathrm{S}$ and the calculated local-electric field supports the notion that the antenna-enhanced field dominates the observed response. Further, nearly identical spatial textures of $\Delta \mathrm{S}$ are observed surrounding a series of four different antennas, at different locations in the same $\mathrm{VO}_{2}$ film, supporting the notion that the antenna-enhanced field is sufficient to overcome other 'background' sources of heterogeneity. A strong enhancement of the local electric field is observed near the top left corner

of each antenna in the simulation in agreement with the enhancement of $\Delta \mathrm{S}$ that is consistently 
observed at all pump-probe time delays after photoexcitation in the experiments (white dashed circle in Fig. 5; see Fig.S5a-c).

Near zero pump-probe time delay, heterogeneity within the film is slight, Fig. S5d-f, consistent with Ref. [S19]. The near-field amplitude near-zero-time delay is, further, directly proportional to the applied pump power (Fig. S5f) ${ }^{\mathrm{S} 20}$ with a nearly-identical response observed at all investigated locations. We remark that the $50 \%$ increase of $\Delta \mathrm{S} / \mathrm{S}$ observed near the resonant antenna is consistent with the $25 \%$ enhancement of the local electric field from our Lumerical simulations in Fig. S5b. Note that in these simulations the magnitude of the electric field away from the antenna is reduced to $\sim 0.8 \mathrm{~V} / \mathrm{m}$.
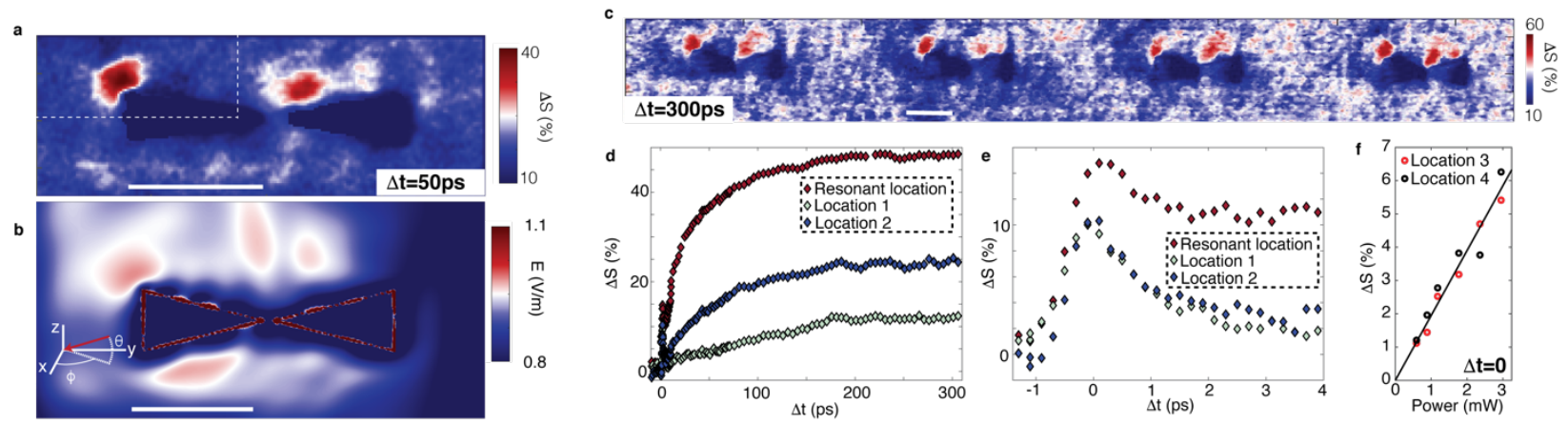

Figure S5: Field enhancement. a, An image of the pump-induced change in near-field amplitude $\Delta \mathrm{S}$ is shown with the false color map. b. The calculated local electric field, simulated at the pump wavelength used in the experiment $(1.03 \mu \mathrm{m})$ as described in the Supplementary text. The three-dimensional schematic shown in the schematic indicates the relevant geometry. The pump is incident along the path marked by the red arrow, which is $\theta=30$ degrees from the sample-normal and $\phi=40$ degrees from the $x$-axis. Note that the calculated image shows a top-down view within the $\mathrm{xy}$ plane, taken at $\mathrm{z}=0$ where the gold antenna and $\mathrm{VO}_{2}$ film intersect. The long axis of the antenna is oriented along $\mathrm{y}$ while the short axis is oriented along $\mathrm{x}$. Note that an incident electric field magnitude of $1 \mathrm{~V} / \mathrm{m}$ is considered in the simulation. c, A large area image of $\Delta \mathrm{S}$ covering four-resonant antenna structures. The scale bars in panels (a)-(c) are $1 \mu \mathrm{m}$ in length. $\mathbf{d}$, and $\mathbf{e}$, Delay traces showing $\Delta \mathrm{S}$ vs. $\Delta \mathrm{t}$ at three locations in the film. The $\Delta \mathrm{S}$ data shown with blue and green diamonds were collected in the interior of the film at two representative locations while the $\Delta \mathrm{S}$ data shown with red dots were collected within the antenna enhanced region. $f$, Data showing the dependence of the $\Delta \mathrm{S}$ on the photo-excitation power near $\Delta \mathrm{t}=0$ at two representative locations within the interior of the thin film. 
Supplementary References:

S1. Liu, M.; Sternbach, A. J.; Wagner, M.; Slusar, T. V.; Kong, T.; Bud'ko, S. L.; Kittiwatanakul, S.; Qazilbash, M. M.; McLeod, A.; Fei, Z.; Abreu, E.; Zhang, J.; Goldflam, M.; Dai, S.; Ni, G.-X.; Lu, J.; Bechtel, H. A.; Martin, M. C.; Raschke, M. B.; Averitt, R. D.; Wolf, S. A.; Kim, H.-T.; Canfield, P. C.; Basov, D. N. Physical Review B 2015, 91, (24), 245155.

S2. Yang, T.-H.; Aggarwal, R.; Gupta, A.; Zhou, H.; Narayan, R. J.; Narayan, J. Journal of Applied Physics 2010, 107, (5), 053514.

S3. Zhao, Y.; Lee, J. H.; Zhu, Y.; Nazari, M.; Chen, C.; Wang, H.; Bernussi, A.; Holtz, M.; Fan, Z. Journal of Applied Physics 2012, 111, (5), 053533.

S4. Kim, C.-Y.; Slusar, T.; Cho, J.; Kim, H.-T. ACS Applied Electronic Materials 2021, 3, (2), 605610.

S5. $\quad$ McLeod, A. S.; Kelly, P.; Goldflam, M. D.; Gainsforth, Z.; Westphal, A. J.; Dominguez, G.; Thiemens, M. H.; Fogler, M. M.; Basov, D. N. Physical Review B 2014, 90, (8), 085136.

S6. $\quad$ Cvitkovic, A.; Ocelic, N.; Hillenbrand, R. Opt. Express 2007, 15, (14), 8550-8565.

S7. Hauer, B.; Engelhardt, A. P.; Taubner, T. Opt. Express 2012, 20, (12), 13173-13188.

S8. Chen, X.; Lo, C. F. B.; Zheng, W.; Hu, H.; Dai, Q.; Liu, M. Applied Physics Letters 2017, 111, (22), 223110.

S9. Abdulhalim, I. Journal of Optics A: Pure and Applied Optics 1999, 1, (5), 646-653.

S10. Rajab, K. Z.; Naftaly, M.; Linfield, E. H.; Nino, J. C.; Arenas, D.; Tanner, D.; Mittra, R.; Lanagan, M. Journal of Microelectronics and Electronic Packaging 2008, 5, (1), 2-7.

S11. Huffman, T. J.; Xu, P.; Qazilbash, M. M.; Walter, E. J.; Krakauer, H.; Wei, J.; Cobden, D. H.; Bechtel, H. A.; Martin, M. C.; Carr, G. L.; Basov, D. N. Physical Review B 2013, 87, (11), 115121.

S12. McLeod, A. S.; van Heumen, E.; Ramirez, J. G.; Wang, S.; Saerbeck, T.; Guenon, S.; Goldflam, M.; Anderegg, L.; Kelly, P.; Mueller, A.; Liu, M. K.; Schuller, I. K.; Basov, D. N. Nature Physics 2017, 13, (1), 80-86.

S13. Chen, Y.; Zhang, S.; Ke, F.; Ko, C.; Lee, S.; Liu, K.; Chen, B.; Ager, J. W.; Jeanloz, R.; Eyert, V.; Wu, J. Nano Letters 2017, 17, (4), 2512-2516.

S14. Park, J. H.; Coy, J. M.; Kasirga, T. S.; Huang, C.; Fei, Z.; Hunter, S.; Cobden, D. H. Nature 2013, 500, (7463), 431-434.

S15. Tselev, A.; Meunier, V.; Strelcov, E.; Shelton, W. A.; Luk'yanchuk, I. A.; Jones, K.; Proksch, R.; Kolmakov, A.; Kalinin, S. V. ACS Nano 2010, 4, (8), 4412-4419.

S16. Wang, Y.; Sun, X.; Chen, Z.; Cai, Z.; Zhou, H.; Lu, T.-M.; Shi, J. Science Advances 2018, 4, (5), eaar3679.

S17. Catalan, G.; Seidel, J.; Ramesh, R.; Scott, J. F. Reviews of Modern Physics 2012, 84, (1), 119-156.

S18. Cahn, J. W. Acta Metallurgica 1956, 4, (5), 449-459.

S19. Sternbach, A. J.; Hinton, J.; Slusar, T.; McLeod, A. S.; Liu, M. K.; Frenzel, A.; Wagner, M.; Iraheta, R.; Keilmann, F.; Leitenstorfer, A.; Fogler, M.; Kim, H. T.; Averitt, R. D.; Basov, D. N. Opt. Express 2017, 25, (23), 28589-28611.

S20. Kübler, C.; Ehrke, H.; Huber, R.; Lopez, R.; Halabica, A.; Haglund, R. F.; Leitenstorfer, A. Physical Review Letters 2007, 99, (11), 116401. 